\title{
The Limnology of Ohana Lake, a Potential Manmade Aquaculture System in Nigeria
}

\author{
Paul O. Ajah \\ Institute of Oceanography, University of Calabar, Calabar, Nigeria \\ Email:ajapaulo@yahoo.com
}

Received February 15, 2013; revised March 18, 2013; accepted March 26, 2013

Copyright (C) 2013 Paul O. Ajah. This is an open access article distributed under the Creative Commons Attribution License, which permits unrestricted use, distribution, and reproduction in any medium, provided the original work is properly cited.

\begin{abstract}
The concentrations of heavy metals $(\mathrm{Fe}>\mathrm{Zn}>\mathrm{Cu}>\mathrm{Pb}>\mathrm{Ag}$ ) in bottom sediments and fish gills in Ohana Lake, were found to be significantly high and far exceeded FEPA and WHO environmental standards for water quality by 1.5 to 18 times, respectively. Six classes of each of phytoplankton and zooplankton with a total of 35 phytoplankton taxa comprising 46 species i.e. 35(46) and 22(28) faunal were observed. The class Chlorophyceae dominated the phytoplankton community with $18(22)$ followed by Cyanobacteria 6(10). The aquatic fauna was dominated by the Rotifera $8(11)$, followed by the Copepoda 6(9). The benthic flora community consisted of five classes of phytoplankton made up of 28(36). The class Bacillariophyceae 11(15) dominated the group followed by Chlorophyceae 10(11). Benthic fauna were made up of seven classes of 13(13). The dominant class Nemata 4(4) was followed closely by Protozoa 2(3). Ohana Lake is fast turning to a eutrophic ecosystem with accompanied algal bloom due to very high nutrient contents. The equitability or evenness indices $(\mathrm{J})$ for both phytoplankton and zooplankton were lowly indicating generally low species diversities as well as predominantly unstable ecosystem. The aquacultural implications of these parameters are discussed.
\end{abstract}

Keywords: Limnology; Aquaculture; Productivity; Pollution; Fishery; Benthos

\section{Introduction}

Residential development of lakeshores is expected to change a variety of key lake features that included increased nutrient loading, increased invasion rate of nonnative species, increased exploitation rates of fishes by anglers, and alteration of littoral habitats. All of these factors may alter the capacity of lakes to support productive native fish populations [1]. Ohana Lake being quite close to residential area witnesses a lot of anthropogenic activities such as bathing; canoeing and car/motorcycle wash, and consequently, is prone to suffer such fate.

Heavy metals are considered as major environmental pollutants and are regarded as being cytotoxic, mutagenic and carcinogenic [2].

Knowledge of the physicochemical regimes of a body of water is invaluable in the determination of the productivity and other characteristics $[3,4]$ opined that fertility of water is related to its chemical properties and an understanding of the water chemistry serves as the basis for determining whether the water is rich or poor in biological production. Physicochemical factors are known to influence vertical and horizontal migration of organisms, the distribution and feeding regime [5]. The water quality of the lakes and rivers is assessed by the physicochemical and biological characteristics of their waters. It is known that several water quality parameters if not within normal range could act as stressors and adversely affect fish growth and reproduction [6]. Hence, regular monitoring of physicochemical factors is essential for assessing the status of lakes with references to fish culture $[7,8]$ noted that density and diversity of flora and fauna were dependent on chemical regime of water. Fish yields from lakes and reservoirs are known to have strong correlation with primary production $[9,10]$. [11] studied the concentration of heavy metals in water and Hemichromis fasciatus of a waste pit influenced by petroleum activities and found that, seasonally, $\mathrm{Cd}, \mathrm{Cu}, \mathrm{Pb}$, $\mathrm{Mn}, \mathrm{Co}, \mathrm{Cr}, \mathrm{V}$ and $\mathrm{Hg}$ had higher mean values in water during the dry season while $\mathrm{Zn}$, Ni and Fe were higher during the raining season. All heavy metals except $\mathrm{Fe}$ were higher in mean values during the dry season in $H$. fasciatus than during the rainy season.

A survey of the benthos makes it possible to establish if pollution has occurred in recent past and, if so, whether 
toxic or organic in nature. When organic pollution has occurred, the number of species is usually restricted, although the few species present may be present in very high numbers. On the other hand, pollution from toxic substances may eliminate almost all animals present except for a few highly resistant species. A survey of the benthos is, therefore often of more use than an analysis of a water sample, since the water sample represents only one sample taken at one particular point in time and tells you little about the integrated, long-term effects of water quality [12].

The study of Ohana Lake is aimed at assessing its limnology and possible aquacultural potentials. Studies like this make significant contribution to fishery management by identifying potential damage to fish populations, through low oxygen levels, toxic blooms or anthropogenic pollution [13].

\section{Description of the Study Area}

Ohana Lake is located between latitudes $05^{\circ} 57^{\prime \prime} 24^{\prime \prime} \mathrm{N}$ and $05^{\circ} 57^{\prime \prime} 31^{\prime \prime} \mathrm{N}$ and longitudes $008^{\circ} 22^{\prime} 38^{\prime \prime} \mathrm{E}$ and $008^{\circ} 22^{\prime} 46^{\prime \prime} \mathrm{E}$. It is situated barely $30 \mathrm{~m}$ away from the major road linking Calabar and Ikom town and right at the centre of Ohana community in Obrubra Local Government area of Cross River State, Nigeria (see Figure 1).

Ohana Lake could be characterized as an oligotrophic perennial lake due to its nutrient poor status. It is also a limnetic lake due to its lack of rooted vegetation. The Lake covers an area of $3.0 \mathrm{~km}^{2}$. The average depth of the Lake was $4.72 \mathrm{~m}$ with maximum depth of $6.5 \mathrm{~m}$ and minimum $0.3 \mathrm{~m}$ with a water volume of $14.173377 \mathrm{~km}^{3}$. It is of low lying vegetation comprising of shrubs and a few permanent trees surrounding it. Lemna minor dominated aquatic macrophyte.

\section{Materials and Methods}

Ohana Lake was sampled fortnightly in 2005 in the month of March. Sampling locations were gotten using a GPS instrument and the reading converted from longitudes and latitudes to Universal Traverse Mercado (UTM) coordinate system. Various water depth positions were first measured using a calibrated line with a lead and latter confirmed using an echo sounder. Heavy metals were analyzed using atomic absorption spectroscopy (A.A.S) following Unicam 919 Solar System. The fish species were identified using [14] while the mollusks were identified using [15]. Both phytoplankton and zooplankton were identified using [16-18]. Primary production of the lake was determined using light and dark BOD bottles at various depths and days in accordance to [19]. The benthic communities were first fractionated using screens with the following ranges; $0.105 \mathrm{~mm}, 0.50$ $\mathrm{mm}, 0.30 \mathrm{~mm}, 0.50 \mathrm{~mm}$ and $0.80 \mathrm{~mm}$ stacked together and shaken vigorously and severally under a slow flowing tap water and latter identified under a microscope for the smaller groups and direct observation for the macro benthos using the appropriate taxonomic keys. Dissolved oxygen and temperature were analyzed by meter/probe methods [20], nitrate by cadmium reduction/diazotization method following [21]. Phosphate was analyzed by molybdenum blue method (spectrophotometrically) [21]. Total dissolved solids (TDS) and conductivity by meter/ probe methods (3000 $\mathrm{HACH})$; turbidity was by transparency disc while Total suspended solids (TSS) and colour was measured using spectrophotometer (3000 $\mathrm{HACH})$. Water hardness $-\mathrm{Ca}^{2+}, \mathrm{Mg}^{2+}$ - by complexometric (titration) method [20,22].

The Shannon-Wiener's index $\left(\mathrm{H}^{1}\right)$ was adopted in calculating the diversity indices of species (ShannonWiever, 1948) while the Equitability or evenness index (J) was used to compare the $\mathrm{H}^{1}$ index.

\section{Results}

Iron $(\mathrm{Fe})$ values from sediment sample averaged 359.8 $\mathrm{mg} / \mathrm{L}$. Likewise, the average values $10.01 \mathrm{mg} / \mathrm{L}, 10.79$ $\mathrm{mg} / \mathrm{L}, 5.9 \mathrm{mg} / \mathrm{L}$ and $37.42 \mathrm{ml} / \mathrm{L}$, respectively, were realized for lead, copper, silver and zinc in the sediments. Results of analysis of fish gills showed that lead was not detected, the rest metals had the following values: iron$35.17 \mathrm{mg} / \mathrm{L}$, copper $-0.315 \mathrm{mg} / \mathrm{L}$, silver $-0.45 \mathrm{mg} / \mathrm{L}$ and zinc $-3.097 \mathrm{mg} / \mathrm{L}$. The summary of the trace metals of Ohana Lake are represented in Table 1.

Table 2 summarizes the physicochemical parameters and nutrients of Ohana Lake, Nigeria during the sampling periods. The hydrographic data and nutrients in Ohana Lake are shown in Table 3. Temperature ranged from $31.7^{\circ} \mathrm{C}$ to $35.0^{\circ} \mathrm{C}$ with average of $33.12^{\circ} \mathrm{C} \pm 1.01^{\circ} \mathrm{C}$. Ohana Lake exhibited a mild thermal stratification. Both temperature and dissolved oxygen decreased with depth. Dissolved oxygen levels in Ohana Lake ranged from 3.9 to $4.1 \mathrm{mgO}_{2} / \mathrm{L}$ with a mean of $4.01 \pm 0.099 \mathrm{mgO}_{2} / \mathrm{L}$. Figure 2 shows the inverse relationships between dissolved oxygen and depth in metres in Ohana Lake. The $\mathrm{pH}$ of the water ranged from 6.90 to 7.79 with average of

Table 1. The mean values of the trace metals of Ohana Lake, Cross River State, Nigeria on fish and sediment samples.

\begin{tabular}{cccc}
\hline Trace Metals & Sediment & Fish Gill & Av. \pm s.d \\
\hline Iron (ppm) & $359.8 \pm 0.92$ & $35.17 \pm 0.10$ & $197.485 \pm 23$ \\
Lead (ppm) & $10.01 \pm 2.20$ & N.a & $10.01 \pm 1.55$ \\
Copper (ppm) & $10.79 \pm 1.55$ & $0.315 \pm 2.64$ & $5.553 \pm 7.40$ \\
Silver (ppm) & $5.90 \pm 0.10$ & $0.45 \pm 0.00$ & $3.175 \pm 3.85$ \\
Zinc (ppm) & $37.42 \pm 2.44$ & $3.067 \pm 2.55$ & $20.244 \pm 24.30$ \\
\hline
\end{tabular}

N.a $=$ Not available. 


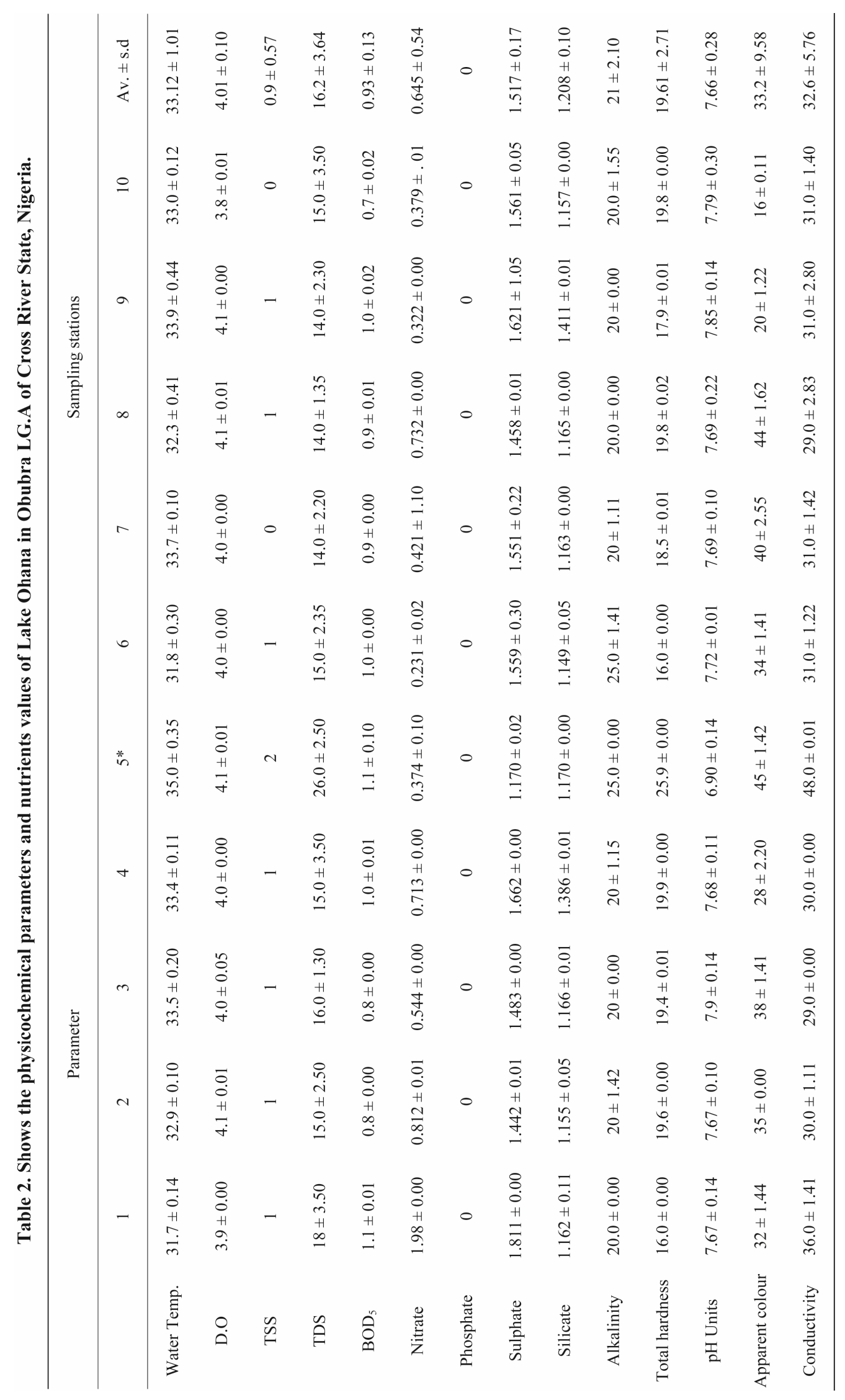




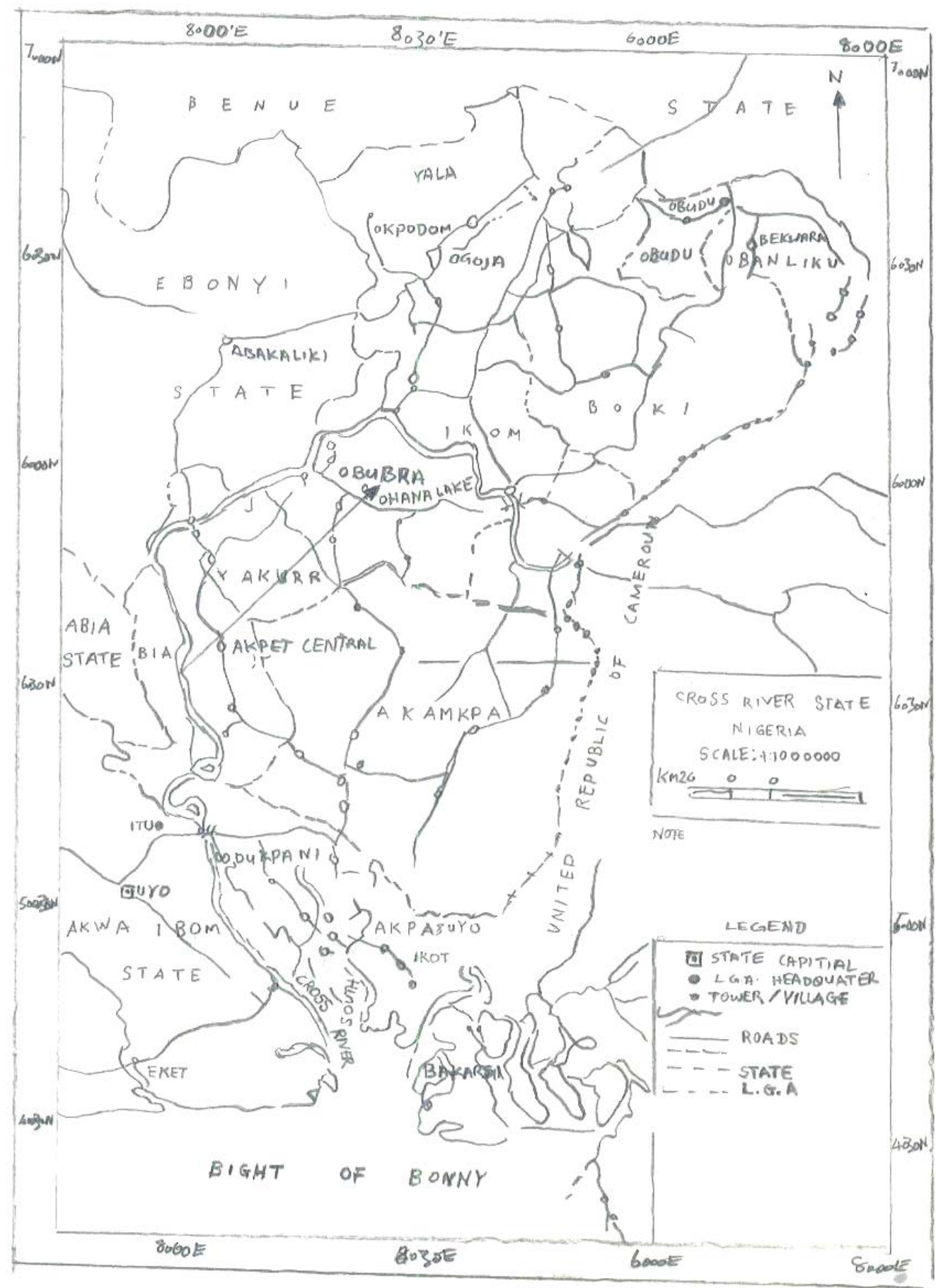

Figure 1. Map of Cross River State, Nigeria, showing the sampling location-Ohana Lake located at Lat. $05^{\circ} 57^{\prime} 24^{\prime} \mathrm{N}$ and $05^{\circ} 57^{\prime} 31^{\prime \prime} \mathrm{N}$ and long. $008^{\circ} 22^{\prime} 38^{\prime \prime} \mathrm{E}$ and $008^{\circ} 22^{\prime} 46^{\prime} \mathrm{E}$ - by an arrow.

$7.66 \pm 0.28$ (Table 3). The total alkalinity in Ohana Lake ranged from 20 to $25 \mathrm{mg} / \mathrm{L}$ with a mean of $21 \pm 2.1 \mathrm{mg} / \mathrm{L}$. The values for total hardness ranged from 16.0 to 25.9 $\mathrm{mg} / \mathrm{l}$ with a mean of $19.61 \pm 2.1$, while that of conductivity was $33.0 \pm 6.46 \mu \mathrm{s} / \mathrm{cm}$ ranging from 29.0 to 48.0 $\mu \mathrm{s} / \mathrm{cm}$. TDS ranged from 14 to 26 with a mean of $16.2 \pm$ 3.64 , nitrate was from 0.231 to $1.98 \mathrm{mg} / \mathrm{l}$ with a mean of
$0.645 \pm 0.54$, sulfate ranged 1.170 to $1.81 \mathrm{mg} / \mathrm{l}$ and averaged $1.517 \pm 0.17$, silicate ranged from 1.149 to 1.386 $\mathrm{mg} / \mathrm{l}$ and had a mean of $1.208 \pm 0.10$ while colour ranged from 28 to $45 \mathrm{pt} / \mathrm{Co}$ with a mean of $33.2 \pm 9.58$.

\section{Plankton Distribution, Density and Diversity}

The water body had six classes each of phytoplankton 
Table 3. Mean hydrographic data of Ohana Lake, Cross River State, Nigeria.

\begin{tabular}{cccc}
\hline Stn & Water Temp. ${ }^{\circ} \mathrm{C}$ & Depth $(\mathrm{m})$ & D.O. $\mathrm{mgO}_{2} / 1$ \\
\hline 1 & $31.7 \pm 0.80$ & $0.3 \pm 0.01$ & $5.5 \pm 1.10$ \\
2 & $32.9 \pm 1.34$ & $1.3 \pm 0.10$ & $3.2 \pm 1.25$ \\
3 & $33.5 \pm 1.23$ & $1.8 \pm 1.20$ & $3.3 \pm 0.08$ \\
4 & $33.4 \pm 1.24$ & $2.0 \pm 0.04$ & $3.4 \pm 1.18$ \\
5 & $35.0 \pm 0.15$ & $2.5 \pm 1.10$ & $3.6 \pm 2.21$ \\
6 & $31.8 \pm 1.35$ & $4.3 \pm 0.00$ & $3.3 \pm 1.16$ \\
7 & $33.7 \pm 1.14$ & $4.5 \pm 2.12$ & $3.4 \pm 1.90$ \\
8 & $32.3 \pm 0.82$ & $5.0 \pm 0.08$ & $3.5 \pm 2.24$ \\
9 & $33.9 \pm 2.02$ & $6.6 \pm 2.10$ & $3.5 \pm 3.15$ \\
10 & $31.7 \pm 1.76$ & $3.8 \pm 0.19$ & $3.4 \pm 1.10$ \\
\hline
\end{tabular}

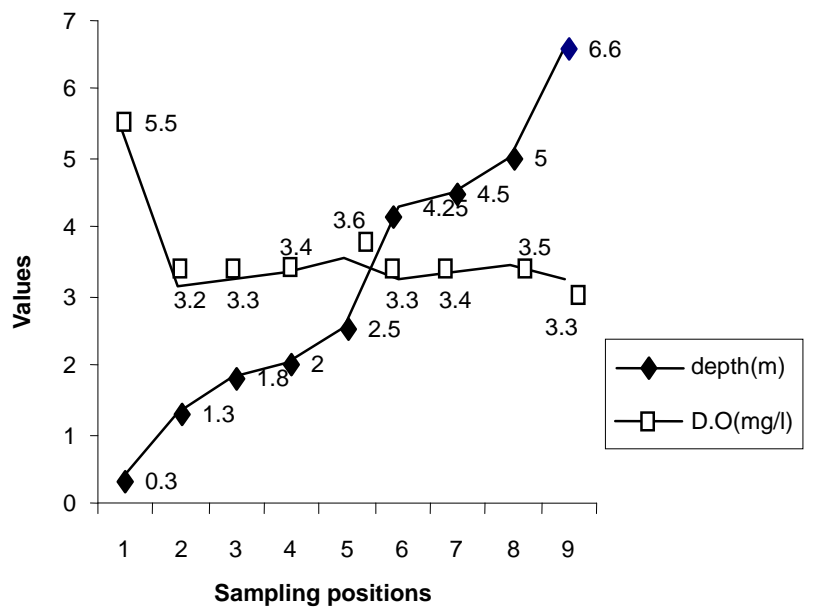

Figure 2. Relationship between depth and dissolved oxygen in Ohana Lake in Cross River State, Nigeria.

and zooplankton with a total of thirty-five phytoplankton taxa comprising forty-six species and twenty-two faunal taxa of twenty-eight species. The class Chlorophyceae dominated the phytoplankton community with eighteen taxa having twenty-two species followed by Cyanobacteria had six taxa comprising ten species and Bacillariophyceae with six taxa and seven species. Both Pyrrophyceae and Xeminophyceae had three taxa and three species each while Euglenophyceae had one species from one taxon. The zooplankton was dominated by Rotifera of eleven species from eight taxa, followed by the Copepoda with six taxa comprising nine species. Both Cladocera and Ostracoda came third in abundance having three taxa and three species each, and lastly Protozoa with two taxon and two species (Table 4).

The benthic flora had five classes of phytoplankton made up of twenty-eight (28) taxa and thirty-six (36) species. The class Bacillariophyceae dominated the group with eleven (11) taxa of fifteen (15) species followed by Chlorophyceae with eleven species from 10 taxa. Cyanobacteria followed this with six (6) taxa and eight (8) species (Table 4). The other two classes Euglenophyceae and Xeminophyceae each had one taxon and one species. The aquatic benthic fauna represented by seven classes of 13 taxa and 13 species was dominated by the class Nemata of 4(4) followed by Protozoa 2(3), Rotifera 2(2), while Bivalvia (Protobranchia); Cladocera, Gasterotricha, Odonata and Ostracoda had one taxon and one species each.

The mean diversity index for the water flora of Ohana Lake was $0.969 \pm 2.55$ and $0.778 \pm 1.23$ for zooplankton. The equitability or evenness index $(\mathrm{J})$ yielded 1.611 and -3.05 , respectively for phytoplankton and zooplankton (Table 3). These low diversity indices indicate generally low species diversities as well as predominantly unstable ecosystem. The diversity index for the benthic flora was $0.371 \pm 0.57$ while that of the benthic fauna was $2.23 \pm$ 2.80 . The equitability or evenness index $(J)$ resulted in -15.50 and 4.18 , respectively, for benthic flora and fauna. The very low floral diversity index values indicate very low species diversities as well as predominantly unstable ecosystem while the faunal diversity indexes indicate slightly higher species diversity and a mild stable ecosystem.

The planktonic community present in the water column of Ohana Lake included the following: Bacillariophyceae (Coscinodiscus excentricus, Ehr, C. lacustris Grunow, Cyclotella stelligera 1 et Grun, Fragilaria sp. Melosira ambigua (Grun), Surirella robusta var splendid, Thermalis denticum), Chlorophyceae (Chlorella vulgaris Beij, Chlamydomonas sp, Chodatella wratislawiensis (Shroeder), Coeolosphaerium dubium, Cosmocladium saxonicum De Bary, Crucigenia rectangularia (Näg.), Eudorina elegans Ehr., Lauterboniella elegantissima Schmidle, Lyngbya limnetica Lemm., Pediastrium sturmii Reinsch, Protococcus sp, Scenedesmus bijuga (Turp.), S. obliquus (Turp.), S. quadricauda Bréb, Spirogyra sp, Staurastrum apiculatum Bréb, Tetradesmus wisconsinensis Smith, Tetrastrum elegans Playfair, T. heterocanthum (Nordst.), Tetraedron tumidulum Hansg, T. minimum (A.Br.), Zygnema sp, Cyanobacteria (Anabaena affinis Lemm, Aphanothece clathrata W. et G.S. West, Aphanothece stagnima B. Peters \& Geitl, Coelosphaerium kuetzingianum Näg, Gloeocapsa limnetica (Lemm.), Merismopedia punctata Meyen, Microcystis aeruginosa Kurz, Microcystis pulverea (Wood), Microcystis aeruginosa F. Flos-aquae, Microcystis Grevillei Hass,), Euglenophyceae Euglena caudate Hübner), Pyrrophyceae (Glenodinium cinctum Ehr, Gymnodinium excavatum Nyg, Peridinium cinctum (O.F.M), Xeminophyceae (Tribonema minus Hazen, Tribonema viride Pasch, 
Table 4. Shows plankton and benthos distribution, density, percentage composition and diversity indices at the given sampling stations in Ohana Lake.

Stn 1

\begin{tabular}{|c|c|c|c|c|c|}
\hline Class & Species & Qty per mL & Density/L (c.75 mLs) & $\%$ Composition & Diversity Index (H') \\
\hline \multirow[t]{5}{*}{ Cyanobacteria } & Microcystis aeruginosa Kurz & 20 & 15 & 51.28 & 2.80 \\
\hline & I (Wood) & 8 & 6 & 20.51 & 0.45 \\
\hline & Microcystis aeruginosa F.Flos-aquae & 8 & 6 & 20.51 & 0.45 \\
\hline & Aphanothece clathrata W. et G.S. West & 1 & 0.75 & 2.56 & 0.007 \\
\hline & Coelosphaerium kuetzingianum Näg & 2 & 1.5 & 5.13 & 0.028 \\
\hline \multirow[t]{3}{*}{ Chlorophyceae } & Chlamydomonas sp & 1 & 0.75 & 14.29 & 0.07 \\
\hline & Pediastrium sturmii Reinsch & 3 & 1.5 & 42.86 & 0.66 \\
\hline & Spirogyra sp & 3 & 2.25 & 42.86 & 0.66 \\
\hline \multicolumn{6}{|l|}{ Zooplankton } \\
\hline \multirow[t]{5}{*}{ Copepoda } & Eudiaptomus gracilis & 2 & 1.5 & 25 & 0.24 \\
\hline & Thermocyclops crassus & 2 & 1.5 & 25 & 0.24 \\
\hline & Thermocyclops neglectus Sars & 1 & 0.75 & 12.5 & 0.06 \\
\hline & Microcyclops rubellus & 2 & 1.5 & 25 & 0.24 \\
\hline & Microcyclops varicans Sars & 1 & 0.75 & 12.5 & 0.06 \\
\hline \multirow[t]{4}{*}{ Rotifera } & Brachionus calyciflorus dorcas (Goose) & 1 & 0.6 & 5.88 & 0.02 \\
\hline & Brachionus falcatus Zacharias & 14 & 10.5 & 82.35 & 4.07 \\
\hline & Brachionus potulus potulus & 1 & 0.75 & 5.88 & 0.02 \\
\hline & Lepadella patella Müller & 1 & 0.75 & 5.88 & 0.02 \\
\hline \multirow[t]{2}{*}{ Cladocera } & Chydorus sphaericus (O.F. Muller) & 1 & 0.75 & 50 & 0.72 \\
\hline & Graptoleberis testudinaria Fischer & 1 & 0.75 & 50 & 0.72 \\
\hline Ostracoda & Cyprinotus pellucidus & 1 & 0.75 & 100 & 0 \\
\hline Odonata & Neurocordulis sp. & 1 & 1.5 & 100 & 0 \\
\hline Protozoa & Arcella vulgaris Ehr. & 2 & 1.5 & 100 & 2.89 \\
\hline
\end{tabular}

$\operatorname{Stn} 2$

\begin{tabular}{|c|c|c|c|c|c|}
\hline Class (Phytoplankton) & Species & Qty per $\mathrm{mL}$ & Density/L (c.185 mLs) & $\%$ Composition & Diversity Index (H') \\
\hline \multirow[t]{2}{*}{ Cyanobacteria } & Microcystis Grevillei Hass. & 3 & 5.55 & 60 & 1.12 \\
\hline & Gloeocapsa limnetica (Lemm.) & 2 & 3.7 & 40 & 0.50 \\
\hline \multirow[t]{7}{*}{ Chlorophyceae } & Pediastrium sturmii Reinsch & 2 & 3.7 & 13.33 & 0.10 \\
\hline & Scenedesmus quadricauda Bréb & 1 & 1.85 & 6.67 & 0.03 \\
\hline & Chlorella vulgaris Beij & 1 & 1.85 & 6.67 & 0.03 \\
\hline & Tetradesmus wisconsinensis Smith. & 1 & 1.85 & 6.67 & 0.03 \\
\hline & Eudorina elegans Ehr. & 3 & 5.55 & 20 & 0.22 \\
\hline & Crucigenia rectangularia (Näg.) & 3 & 5.55 & 20 & 0.22 \\
\hline & Protococcus sp & 4 & 7.40 & 26.67 & 0.39 \\
\hline Хетіпорһусеае & Tribonema minus Hazen. & 1 & 1.85 & 100 & 0 \\
\hline Bacillariophyceae & Melosira ambigua (Grun) & 1 & 1.85 & 100 & 0 \\
\hline \multirow[t]{2}{*}{ Pyrrophyceae } & Glenodinium cinctum Ehr. & 7 & 12.95 & 87.5 & 2.95 \\
\hline & Gymnodinium excavatum Nyg. & 1 & 1.85 & 12.5 & 0.06 \\
\hline \multicolumn{6}{|l|}{ Zooplankton } \\
\hline \multirow[t]{4}{*}{ Copepoda } & Bryocamptus birsteinii & 1 & 1.85 & 12.5 & 0.06 \\
\hline & Eudiaptomus gracilis & 1 & 1.85 & 12.5 & 0.06 \\
\hline & Tropodiaptomus protoceifer & 1 & 1.85 & 12.5 & 0.06 \\
\hline & Cop. Nauplii & 5 & 9.25 & 62.5 & 1.50 \\
\hline Rotifera & Brachionus falcatus Zacharias & 1 & 1.85 & 100 & 0 \\
\hline Cladocera & Dunhevedia serrata Daday & 1 & 1.85 & 100 & 0 \\
\hline
\end{tabular}


Stn 3

\begin{tabular}{|c|c|c|c|c|c|}
\hline Class & Species & Qty per mL & Density/L (c.137 mLs) & $\%$ Composition & Diversity Index (H') \\
\hline \multirow[t]{4}{*}{ Cyanobacteria } & Microcystis aeruginosa Kurz & 11 & 15.07 & 57.89 & 2.16 \\
\hline & Merismopedia punctata Meyen & 4 & 5.48 & 21.05 & 0.29 \\
\hline & Gloecapsa limnetica (Lemm.) & 1 & 1.37 & 5.26 & 0.02 \\
\hline & Aphanothece clathrata W. et G.S. West & 3 & 4.11 & 15.79 & 0.16 \\
\hline \multirow[t]{4}{*}{ Chlorophyceae } & Chlorella vulgaris Beij & 3 & 4.11 & 37.5 & 0.54 \\
\hline & Pediastrium sturmii Reinsch & 1 & 1.37 & 12.5 & 0.06 \\
\hline & Lauterboniella elegantissima Schmidle & 1 & 1.37 & 12.5 & 0.06 \\
\hline & Scenedesmus obliquus (Turp.) & 3 & 4.11 & 37.5 & 0.54 \\
\hline \multirow[t]{2}{*}{ Xeminophyceae } & Tribonema minus Hazen & 2 & 2.74 & 66.67 & 1.21 \\
\hline & Tribonema viride Pasch & 1 & 1.37 & 33.33 & 0.30 \\
\hline \multirow[t]{2}{*}{ Bacillariophyceae } & Coscinodiscus excentricus Her & 2 & 2.74 & 50 & 0.72 \\
\hline & Thermalis denticum & 2 & 2.74 & 50 & 0.72 \\
\hline \multirow[t]{6}{*}{ Copepoda } & Heterocape appendiculata Sars & 1 & 1.37 & 11.11 & 0.051 \\
\hline & Thermocyclops crassus & 2 & 2.74 & 22.22 & 0.20 \\
\hline & Thermocyclops kawamurai Kikuchi & 1 & 1.37 & 11.11 & 0.051 \\
\hline & Bryocamptus birsteinii & 3 & 4.11 & 33.33 & 0.455 \\
\hline & Microcyclops varicans (Sar) & 1 & 1.37 & 11.11 & 0.051 \\
\hline & U.i & 1 & 1.37 & 11.11 & 0.051 \\
\hline \multirow[t]{2}{*}{ Rotifera } & Brachionus calyciflorus dorcas (goose) & 1 & 1.37 & 20 & 0.12 \\
\hline & Brachionus falcatus Zacharias & 4 & 5.48 & 80 & 1.99 \\
\hline \multicolumn{6}{|c|}{ Stn 5} \\
\hline Class & Species & Qty per $\mathrm{mL}$ & Density/L (c.2 mLs) & $\%$ Composition & Diversity Index (H') \\
\hline Cyanobacteria & Microcystis aeruginosa Kurz & 2 & 0.04 & 100 & 0.72 \\
\hline \multirow[t]{12}{*}{ Chlorophyceae } & Pediastrium sturmii Reinsch & 2 & 0.04 & 3.03 & 0.01 \\
\hline & Scenedesmus obliquus (Turp.) & 2 & 0.04 & 3.03 & 0.01 \\
\hline & Scenedesmus quadricauda Bréb & 2 & 0.04 & 3.03 & 0.01 \\
\hline & Scenedesmus bijuga (Turp.) & 6 & 0.12 & 9.09 & 0.13 \\
\hline & Chlorella vulgaris Beij & 29 & 0.58 & 43.94 & 3.04 \\
\hline & Tetradesmus wisconsinensis Smith & 7 & 0.14 & 10.61 & 0.18 \\
\hline & Tetrastrum elegans Playfair & 7 & 0.14 & 10.61 & 0.18 \\
\hline & Eudorina elegans Ehr. & 6 & 0.12 & 9.09 & 0.13 \\
\hline & Tetraedron tumidulum Hansg & 1 & 0.02 & 1.51 & 0.004 \\
\hline & Protococcus sp & 1 & 0.02 & 1.51 & 0.004 \\
\hline & Lyngbya limnetica Lemm. & 2 & 0.04 & 3.03 & 0.01 \\
\hline & Chlamydomonas sp & 1 & 0.02 & 1.51 & 0.004 \\
\hline Xeminophyceae & Tribonema vulgare Pasch & 1 & 0.02 & 100 & 0 \\
\hline Bacillariophyceae & Cyclotella stelligera $\mathrm{Cl}$. et Grun & 3 & 0.06 & 100 & 2.73 \\
\hline Pyrrophyceae & Glenodinium cinctum Her & 18 & 0.36 & 100 & 6.23 \\
\hline Copepoda & Bryocamptus birsteinii & 7 & 0.14 & 100 & 3.60 \\
\hline \multirow[t]{4}{*}{ Rotifera } & Brachionus falcatus Zacharias & 1 & 0.02 & 25 & 0.18 \\
\hline & Brachionus calyciflorus dorcas (Goose) & 1 & 0.02 & 25 & 0.18 \\
\hline & Testudinella caeca & 1 & 0.02 & 25 & 0.18 \\
\hline & Lecane luna Müller & 1 & 0.02 & 25 & 0.18 \\
\hline Cladocera & Alona monachanta (Stingelin) & 1 & 0.02 & 100 & 0 \\
\hline
\end{tabular}


Stn 6

\begin{tabular}{|c|c|c|c|c|c|}
\hline Class & Species & Qty per mL & Density/L (c.112 mLs) & $\%$ Composition & Diversity Index (H') \\
\hline Cyanobacteria & Microcystis aeruginosa Kurz & 2 & 2.24 & 100 & 2.88 \\
\hline \multirow[t]{4}{*}{ Chlorophyceae } & Pediastrium sturmii Reinsch & 1 & 1.12 & 25 & 0.18 \\
\hline & Chlamydomonas sp & 1 & 1.12 & 25 & 0.18 \\
\hline & Spirogyra sp & 1 & 1.12 & 25 & 0.18 \\
\hline & Pleodorina californica Shaw & 1 & 1.12 & 25 & 0.18 \\
\hline \multirow[t]{2}{*}{ Bacillariophyceae } & Coscinodiscus excentricus Ehr & 1 & 1.12 & 50 & 0.72 \\
\hline & Coscinodiscus lacustris Grunow & 1 & 1.12 & 50 & 0.72 \\
\hline Copepoda & Bryocamptus birsteinii & 2 & 2.24 & 100 & 2.88 \\
\hline \multirow[t]{3}{*}{ Rotifera } & Ascomorpha ecudis & 1 & 1.12 & 12.5 & 0.06 \\
\hline & Brachionus falcatus Zacharias & 6 & 6.72 & 75 & 2.16 \\
\hline & Brachionus potulus potulus & 1 & 1.12 & 12.5 & 0.06 \\
\hline Cladocera & Chydorus sphaericus (O.F. Muller) & 1 & 1.12 & 100 & 0 \\
\hline Ostracoda & Physiocypria inflate & 1 & 1.12 & 100 & 0 \\
\hline \multicolumn{6}{|c|}{$\operatorname{Stn} 8$} \\
\hline Class & Species & & Qty per mL & $\mathrm{ty} / \mathrm{L}(\mathrm{c} .60 \mathrm{mLs})$ & Diversity Index(D') \\
\hline \multirow[t]{2}{*}{ Cyanobacteria } & Microcystis aeruginosa $\mathrm{K}$ & & 1 & 0.6 & 0.30 \\
\hline & Aphanothece stagnima B. Peter & Geitl & 2 & 1.2 & 1.21 \\
\hline Bacillariophyceae & Coscinodiscus excentricus & & 1 & 0.6 & 0 \\
\hline \multirow[t]{3}{*}{ Rotifera } & Brachionus calyciflorus dorcas & joose) & 1 & 0.6 & 0.03 \\
\hline & Brachionus falcatus Zach & & 10 & 6 & 3.35 \\
\hline & Brachionus diversicorn & & 1 & 0.6 & 0.03 \\
\hline Cladocera & Leyaigia macrodonta $\mathrm{S}$ & & 1 & 0.6 & 0 \\
\hline \multirow[t]{2}{*}{ Ostracoda } & Cyprinotus pelucidus & & 1 & 0.6 & 0.30 \\
\hline & Cyst & & 2 & 1.2 & 1.21 \\
\hline Protozoa & Stentor polymorphus Mü & & 3 & 1.8 & 2.73 \\
\hline
\end{tabular}

Stn 10

\begin{tabular}{|c|c|c|c|c|c|}
\hline Class & Species & Quantity per $\mathrm{mL}$ & Density/L (c.131 mLs) & \% Composition & Diversity Index (H') \\
\hline \multirow[t]{3}{*}{ Cyanobacteria } & Microcystis Grevillei Hass. & 1 & 1.31 & 2.56 & 0.01 \\
\hline & Aphanothece clathrata W. et G.S. West & 28 & 36.68 & 71.79 & 5.49 \\
\hline & A. stagnina Peter \& Geitl & 10 & 13.10 & 25.64 & 0.70 \\
\hline \multirow[t]{4}{*}{ Chlorophyceae } & Scenedesmus quadricauda Bréb & 1 & 1.31 & 1.04 & 0.002 \\
\hline & Chlorella vulgaris Beij & 92 & 120.52 & 95.83 & 19.32 \\
\hline & Coeolosphaerium dubium & 1 & 1.31 & 1.04 & 0.002 \\
\hline & Cosmocladium saxonicum De Bary & 2 & 2.62 & 2.08 & 0.009 \\
\hline Xeminophyceae & Tribonema minus Hazen & 1 & 1.31 & 100 & 0 \\
\hline Bacillariophyceae & Surirella robusta var splendida & 1 & 1.31 & 100 & 0 \\
\hline Pyrrophyceae & Glenodinium cinctum Ehr. & 4 & 5.24 & 100 & 2.88 \\
\hline Copepoda & Tropodiaptomus protoceifer & 5 & 6.55 & 100 & 3.11 \\
\hline \multirow[t]{2}{*}{ Ostracoda } & Entocythere talulus Hoff & 1 & 1.31 & 50 & 0 \\
\hline & Cyprinotus pellucidus & 1 & 1.31 & 50 & 0 \\
\hline \multirow[t]{3}{*}{ Cladocera } & Alona rectangula rectangular Sars & 1 & 1.31 & 25 & 0.18 \\
\hline & Dunhevedia crassa King & 1 & 1.31 & 25 & 0.18 \\
\hline & Cladocera nauplii & 2 & 2.62 & 50 & 0.72 \\
\hline \multirow[t]{6}{*}{ Rotifera } & Lecane ungulate & 62 & 81.22 & 32.63 & 3.85 \\
\hline & Nothommata aurita & 1 & 1.31 & 0.53 & 0.001 \\
\hline & Monostylla bulla & 56 & 73.36 & 29.47 & 3.15 \\
\hline & Asplanchna priodonta & 52 & 68.12 & 27.37 & 2.71 \\
\hline & Brachionus falcatus Zacharias & 9 & 11.79 & 4.74 & 0.08 \\
\hline & Rotifer cysts & 10 & 13.1 & 5.26 & 0.10 \\
\hline
\end{tabular}


Benthos within $0.105 \mathrm{~mm}$ Sieve

\begin{tabular}{|c|c|c|c|c|c|}
\hline Class & Species & Qty per mL & Density/L (c.130 mLs) & $\%$ Composition & Diversity Index (H') \\
\hline \multirow[t]{9}{*}{ Chlorophyceae } & Scenedesmus armatus (Chod.) & 2 & 2.60 & 16.66 & 0.13 \\
\hline & Scenedesmus bijuga (Turp.) & 1 & 1.30 & 8.3 & 0.03 \\
\hline & $\begin{array}{c}\text { Closteriopsis longissima var. tropica } \\
\text { W. \& G. west }\end{array}$ & 1 & 1.30 & 8.3 & 0.03 \\
\hline & Closterium strigosum Bréb & 1 & 1.30 & 8.3 & 0.03 \\
\hline & Eudorina elegans Ehr. & 1 & 1.30 & 8.3 & 0.03 \\
\hline & Tetradesmus wisconsinensis Smith. & 1 & 1.30 & 8.3 & 0.03 \\
\hline & Tetrastrum heterocanthum (Nordst.) & 3 & 3.9 & 23.07 & 0.30 \\
\hline & Pediastrum boryanum Menegh. & 1 & 1.30 & 8.3 & 0.03 \\
\hline & Spirogyra sp & 1 & 1.30 & 8.3 & 0.03 \\
\hline \multirow[t]{5}{*}{ Bacillariophyceae } & Surirela robusta var.splendida & 2 & 1.30 & 28.57 & 0.29 \\
\hline & Nitzschia sigma (Kürtz) & 1 & 1.30 & 14.29 & 0.07 \\
\hline & Achnanthes lanceolta & 2 & 2.60 & 28.57 & 0.29 \\
\hline & Pinnularia Braunii & 1 & 1.30 & 14.29 & 0.07 \\
\hline & Gomphonema sphaerophorum & 1 & 1.30 & 14.29 & 0.07 \\
\hline \multirow[t]{2}{*}{ Cyanobacteria } & Anabaena sp. & 1 & 1.30 & 50 & 0.72 \\
\hline & Oscillatoria limosa Ag. & 1 & 1.30 & 50 & 0.72 \\
\hline Xeminophyceae & Tribonema minus Hazen & 1 & 1.30 & 100 & 0 \\
\hline \multirow[t]{2}{*}{ Nemata } & Rhabdolaimus minors & 3 & 3.90 & 60 & 1 \\
\hline & Trilobus longus & 2 & 2.60 & 40 & 0.50 \\
\hline \multirow[t]{2}{*}{ Protozoa } & Chilodonella spp & 13 & 16.90 & 92.86 & 4.57 \\
\hline & Paramecium caudatum Ehr. & 1 & 1.30 & 7.14 & 0.027 \\
\hline Gasterotricha & Chaetonotus sp & 1 & 1.30 & 100 & 0 \\
\hline
\end{tabular}

Benthos within $0.150 \mathrm{~mm}$ Sieve

\begin{tabular}{|c|c|c|c|c|c|}
\hline Class (Flora) & Species & Qty per $\mathrm{mL}$ & Density/L (c.200 mLs) & $\%$ Composition & Diversity Index (H') \\
\hline Xeminophyceae & Tribonema minus Hazen & 1 & 2 & 100 & 0 \\
\hline Euglenophyceae & Euglena oxyuris Schmarda. & 1 & 2 & 100 & 0 \\
\hline \multirow[t]{3}{*}{ Bacillariophyceae } & Denthicum thermalis & 1 & 2 & 20 & 0.12 \\
\hline & Nitzschia sigma (Kürtz) & 2 & 4 & 40 & 0.50 \\
\hline & Coscinodiscus excentricus Ehr. & 2 & 4 & 40 & 0.50 \\
\hline \multirow[t]{4}{*}{ Cyanobacteria } & Aphanothece stagnina B. Peters et Geitl. & 2 & 4 & 15.38 & 0.12 \\
\hline & Microcystis sp & 1 & 2 & 11.11 & 0.03 \\
\hline & Dactylococcopsis irregularis Smith & 9 & 18 & 69.23 & 2.43 \\
\hline & Spirulina princeps B. Peters et Geitl. & 1 & 2 & 11.11 & 0.03 \\
\hline \multicolumn{6}{|l|}{ Class (Fauna) } \\
\hline Nemata & Rhabdolaimus minors & 3 & 6 & 100 & 2.73 \\
\hline Rotifera & Philodina erythrophthalma Ehr. & 2 & 4 & 100 & 2.88 \\
\hline
\end{tabular}


Benthos within $0.30 \mathrm{~mm}$ Sieve

\begin{tabular}{|c|c|c|c|c|c|}
\hline Class (Flora) & Species & Qty per mL & Density/L (c.200 mLs) & $\%$ Composition & Diversity Index (H') \\
\hline Chlorophyceae & Eudorina elegans Ehr. & 1 & 2 & 100 & 0 \\
\hline \multirow[t]{6}{*}{ Bacillariophyceae } & Achnantes inflata & 8 & 16 & 53.33 & 1.58 \\
\hline & Pinnularia appendiculata (Ag.) & 1 & 2 & 6.66 & 0.02 \\
\hline & Coscinodiscus excentricus Ehr & 3 & 6 & 20 & 0.22 \\
\hline & Cymbella naviculiformis Auerswald. & 1 & 2 & 6.66 & 0.02 \\
\hline & Surirella Capronii Bréb & 1 & 2 & 6.66 & 0.02 \\
\hline & Stauroneis anceps Ehr. & 1 & 2 & 6.66 & 0.02 \\
\hline \multirow[t]{3}{*}{ Cyanobacteria } & Aphanothece stagnina B. Peters et Geitl. & 1 & 2 & 20 & 0.12 \\
\hline & Microcystis Grevillei & 2 & 4 & 40 & 0.50 \\
\hline & Spirulina princes B. Peters et Geitl. & 2 & 4 & 40 & 0.50 \\
\hline \multicolumn{6}{|l|}{ Class (Fauna) } \\
\hline Gasterotricha & Chaetonotus sp & 2 & 4 & 100 & 2.88 \\
\hline Rotifera & Eothinia elongate Ehr. & 1 & 2 & 100 & 0 \\
\hline Protozoa & Chilodonella uncinata & 13 & 26 & 100 & 5.06 \\
\hline \multirow[t]{2}{*}{ Nemata } & Aphelenchoide microlaimus & 3 & 6 & 50 & 0.84 \\
\hline & Rhabdolaimus minors & 3 & 6 & 50 & 0.84 \\
\hline
\end{tabular}

Benthos within $0.40 \mathrm{~mm}$ Sieve

\begin{tabular}{cccccc}
\hline Class (Flora) & Species & Quantity per mL & Density/L (conc.60 mLs) & \% Composition & Diversity Index (H') \\
\hline Chlorophyceae & Paramecium caudatum Ehr. & 1 & 0.6 & 11.11 & 0.05 \\
& Lyngbya limnetica Lemm. & 1 & 0.6 & 11.11 & 0.05 \\
& Closterium strigosum Bréb. & 6 & 3.6 & 66.66 & 1.82 \\
& Hyalotheca dissiliens (Sm.) & 1 & 0.6 & 11.11 & 0.05 \\
Cyanobacteria & Aphanothece clathrata W. et G.S. West & 1 & 0.6 & 20 & 0.12 \\
& Microcystis Grevillei Hass. & 1 & 0.6 & 60 & 0.12 \\
Class (Fauna) & Spirulina princeps B. Peters et Geitl. & 3 & 1.8 & & 1.12 \\
Cladocera & & & & 100 & 0 \\
Ostracoda & Chydorus sphaericus & 1 & 0.6 & 100 & 0 \\
Rotifera & Physiocypria inflata & 1 & 0.6 & 100 & 0 \\
Protozoa & Eothinia elongate Ehr. & 2 & 1.2 & 100 & 2.88 \\
Nemata & Chilodonella uncinata & 22 & 13.2 & 50 & 7.11 \\
& Rhabdolaimus minors & 2 & 1.2 & 50 & 0.72 \\
\hline
\end{tabular}

Benthos within $0.50 \mathrm{~mm}$ Sieve

\begin{tabular}{cccccc}
\hline Class (Flora) & Species & Qty per mL & Density/L (c.190 mLs) & \% Composition & Diversity Index (H') \\
\hline Chlorophyceae & Closterium strigosum Bréb & 5 & 9.50 & 71.43 & 1.84 \\
& Scenedesmus armatus (Chod.) & 2 & 3.80 & 28.57 & 0.29 \\
Cyanobacteria & Aphanothece clathrata W. et G.S. West & 5 & 9.50 & 71.43 & 1.84 \\
& Spirulina princeps B. Peters et Geitl. & 2 & 3.80 & 28.57 & 0.29 \\
Bacillariophyceae & Achnanthes Peragallii & 1 & 1.90 & 16.66 & 0.09 \\
& Coscinodiscus excentricus Ehr & 1 & 1.90 & 33.33 & 0.09 \\
Class (Fauna) & Gyrosigma acuminatum (Kürtz.) & 2 & 3.80 & 3.33 & 0.37 \\
Nemata & Surirella robusta Ehr. & 2 & & & 0.37 \\
Protozoa & Aphelenchoide microlaimus & 5 & 9.80 & 100 & 3.11 \\
& Chilodonella uncinata & 1 & 7.90 & 2.38 & 0.11 \\
\hline
\end{tabular}


Benthos within $0.8 \mathrm{~mm}$ Sieve

\begin{tabular}{ccccc}
\hline Class (Fauna) & Species & Qty per $\mathrm{mL}$ & \% Composition & Diversity Index (H') \\
\hline Bivalvia (Probranchia) & Nucula sulcata Bonn & 2 & 100 & 2.88 \\
\hline
\end{tabular}

Tribonema vulgare Pasch) while the Zooplankton community included: Copepoda (Bryocamptus birsteinii, Eudiaptomus gracilis, Heterocape appendiculata Sars, Microcyclops rubellus, Microcyclops varicans Sars, Thermocyclops crassus, Thermocyclops kawamurai Kikuchi, Thermocyclops neglectus Sars, Tropodiaptomus protoceifer, Cop. Nauplii), Protozoa (Arcella vulgaris Ehr., Stentor polymorphus Müller), Rotifera (Ascomorpha ecudis, Asplanchna priodonta, Brachionus calyciflorus dorcas (Goose), Brachionus falcatus Zacharias, Brachionus potulus potulus, Lecane luna Müller, Lecane ungulate, Lepadella patella Müller, Monostylla bulla, Nothommata aurita, Rotifer cysts, Testudinella caeca,) Cladocera (Chydorus sphaericus (O.F. Muller), Graptoleberis testudinaria Fischer, Dunhevedia serrata Daday, and Ostracoda (Cyprinotus pellucidus, Entocythere talulus Hoff, Physiocypria inflate)

Similarly the benthic floral and faunal of Ohana Lake consisted of Bacillariophyceae (Achnanthes inflate, A. lanceolta, A. Peragallii, Coscinodiscus excentricus Ehr, Cymbella naviculiformis Auerswald, Denthicum thermalis, Gomphonema sphaerophorum, Gyrosigma acuminatum (Kürtz.), Nitzschia sigma (Kürtz), Pinnularia Braunii, P. appendiculata (Ag.), Stauroneis anceps Ehr, Surirella Capronii Bréb, Surirella robusta Ehr., Surirella robusta var. splendida), Chlorophyceae (Closteriopsis longissima var. tropica W. \& G. west, Closterium strigosum Bréb, Eudorina elegans Ehr., Hyalotheca dissiliens (Sm.), Lyngbya limnetica Lemm., Pediastrum boryanum Menegh., Scenedesmus armatus (Chod.), S. bijuga (Turp.), Spirogyra sp Tetradesmus wisconsinensis Smith. Tetrastrum heterocanthum (Nordst.), Cyanobacteria (Anabaena sp., Aphanothece clathrata W. et G.S. West, A. stagnina B. Peters et Geitl., Dactylococcopsis irregularis Smith, Microcystis Grevillei, Microcystis sp., Oscillatoria limosa Ag., Spirulina princeps B. Peters et Geitl.,) Euglenophyceae (Euglena oxyuris Schmarda.), Xeminophyceae (Tribonema minus Hazen)

The Benthic fauna species diversity in Ohana Lake Included Cladocera (Chydorus sphaericus (O.F.Muller), Bivalvia (Nucula sulcata Bonn), Gasterotricha (Chaetonotus sp), Nemata (Aphelenchoide microlaimus, Diplogaster factor, Rhabdolaimus minors, Trilobus longus), Ostracoda (Physiocypria inflate), Odonata (Neurocordulis sp.), Protozoa (Chilodonella spp Chilodonella uncinata, Paramecium caudatum Ehr,), and Rotifera (Eothinia elongate Ehr. Philodina erythrophthalma Ehr.).

The net photosynthetic activity ranged from $-0.0312 \mathrm{C}$ in $\mathrm{mg} / \mathrm{L}$ to $0.312 \mathrm{C}$ in $\mathrm{mg} / \mathrm{L}$ with a mean of $-0.063 \pm$
$0.277 \mathrm{C}$ in $\mathrm{mg} / \mathrm{L}$ while the gross photosynthetic activity ranged from 0.0 joules $(\mathrm{j})$ to $23.13 \mathrm{j}$ with a mean of $7.44 \pm$ $10.50 \mathrm{j}$. Net primary production at surface $(0.0 \mathrm{~m})$, middle $(0.5 \mathrm{~m})$ and $1.0 \mathrm{~m}$ depth, respectively, for four samplings were $-0.0312 \mathrm{C}$ in $\mathrm{mg} / \mathrm{L},-0.412 \mathrm{C}$ in $\mathrm{mg} / \mathrm{L},-0.686 \mathrm{C}$ in $\mathrm{mg} / \mathrm{L} ;-0.312 \mathrm{C}$ in $\mathrm{mg} / \mathrm{L}, 0.094 \mathrm{C}$ in $\mathrm{mg} / \mathrm{L}, 0.094 \mathrm{C}$ in $\mathrm{mg} / \mathrm{L} ; 0.062 \mathrm{C}$ in $\mathrm{mg} / \mathrm{L}, 0.062 \mathrm{C}$ in $\mathrm{mg} / \mathrm{L},-0.125 \mathrm{C}$ in $\mathrm{mg} / \mathrm{L}$; and $0.312 \mathrm{C}$ in $\mathrm{mg} / \mathrm{L}, 0.094 \mathrm{C}$ in $\mathrm{mg} / \mathrm{L}, 0.094 \mathrm{C}$ in $\mathrm{mg} / \mathrm{L}$. The corresponding Gross photosynthetic activity were 10.28 energy in joules $(\mathrm{j}), 3.855 \mathrm{j}, 0.0 \mathrm{j} ; 2.57 \mathrm{j}, 23.13 \mathrm{j}$, $10.28 \mathrm{j} ; 2.57 \mathrm{j}, 2.57 \mathrm{j},-14.778 \mathrm{j}$; and $15.42 \mathrm{j}, 23.13 \mathrm{j}, 10.28 \mathrm{j}$. Positive correlation $(\mathrm{r}=0.504)$ was observed between net and gross photosynthetic activity in Ohana Lake. Decreasing photosynthetic activity was observed when the light and dark bottles were allowed to stay beyond the period of active photosynthesis (10 a.m. to 4.00 p.m.).

\section{Discussion}

The concentration of heavy metals in the fish gills and bottom sediments from Ohana Lake were found to be significantly high and exceeded the water quality criteria by $1 \frac{1}{2}$ to 18 times, respectively. Besides lead that was not detected in fish, iron, copper, silver and zinc all exceeded the [23] set standards in both fish and sediments. Iron values were beyond the $20 \mathrm{mg} / \mathrm{L}$ upper limit for polluted environment [23-25]), which puts Ohana Lake as a highly polluted environment. Comparatively, the concentration of heavy metals in the interstitial water was found to be significantly high and exceeded the water quality criteria by three to eleven times [26]. [11] opined that the mean abundance of heavy metals in $H$. fasciatus was $\mathrm{Fe}>\mathrm{Zn}>\mathrm{Mn}>\mathrm{Cu}>\mathrm{Cd}>\mathrm{Co}>\mathrm{Pb}>\mathrm{Ni}>$ $\mathrm{Cr}>\mathrm{V}$. The monthly concentrations of heavy metals in $H$. fasciatus were far higher than those in water. The concentrations of $\mathrm{Pb}, \mathrm{Fe}$ and $\mathrm{Mn}$ were above WHO limits for drinking water for some months while Fe and $\mathrm{Mn}$ values in $H$. fasciatus were above WHO limits for food in twelve months with $\mathrm{Zn}, \mathrm{Cd}, \mathrm{Cu}, \mathrm{Pb}, \mathrm{Ni}$, $\mathrm{Co}$ and $\mathrm{Cr}$ exceeding WHO limits for some months. The findings reveal that the water and the fish (H. fasciatus) are contaminated and not fit for drinking and consumption by humans. The heavy metal pollution of natural environment has been consistently increasing through effluents, sedimentation of rocks and mining activities [2]. The high load of heavy metals in Ohana Lake must have been due to accumulation of heavy metals from the explosives used during quarrying activities and sedimentation of rocks and wash off from cars etc. [27] indicated that the general pattern in descending order of heavy metals 
accumulation in the muscle of the fishes examined is $\mathrm{Fe}$ $>\mathrm{Zn}>\mathrm{Cu}>\mathrm{Pb}$. The highest mean value of heavy metals recorded is the iron level in Scomber scombrus (944.38+ $548.58 \mathrm{mg} / \mathrm{kg}$ ). The concentration of iron and copper were significantly different $(\mathrm{P}<0.05)$ among the fish samples. The mean value of Iron in Scomber scombrus samples examined was higher than the World Health Organization (WHO) allowable limit in food.

The presence of metal pollutants in freshwater is known to disturb the balance of aquatic ecosystem and this has been noticed to manifest in the presence of some irregularities in fish physiology as fishes tend to concentrate some metals in their body tissues [28]. It was also recognized that the increased environmental burdens of metals and acids in lakes were potentially stressful to local fisheries. For instance, fish population may be lost from lake apparently because of reproductive failures [29]. Thus, fishes are sensitive indicators of heavy metals pollution [30]. Heavy metals such as lead, cadmium, zinc, iron, silver, mercury and chromium etc have not been shown to be essential for life. Rather they are introduced into our environment in large quantities by human activities. Organometalic derivatives of these metals are usually volatile and may be concentrated in the fatty tissues, and sometimes result in chromosomal damage thus making them particularly dangerous. [31] noted that the use of fish alone to access the level of heavy metals may be misleading if not carefully interpreted.

The temperature range of Ohana Lake fell within the recommended temperature conducive for optimum growth of fish in the tropics according to the Institute of Food and Agriculture Sciences, University of Florida (IFAS, Circular 1051). The study shows that the lake water temperature remained around the upper limit of the optimum range throughout the sampling periods and hence might not probably affect fish growth. However, [32] observed a milder water temperature in Lake Chad to be $24.63^{\circ} \mathrm{C} \pm 4.70^{\circ} \mathrm{C}$, which must have contributed to the high productive nature of Lake Chad compared to the low productive nature of Ohana Lake.

The dissolved oxygen level in Ohana Lake was lower than that of Lake Chad $\left(6.95 \pm 0.53 \mathrm{mgO}_{2} / \mathrm{L}\right)$ [32]. Low DO level, indicates decomposition of organic matter and bacterial and may be caused by high temperature leading to decreased oxygen holding capacity of water as suggested by [33] for Senthil sarovar and due to higher trophogenic activities as observed in Lake Taudaha in India [34]. The minimum DO level required in a lake to support average or good fish production is $>5 \mathrm{ppm}$ [35]. Both the range and mean DO in Ohana Lake fell below the minimum, and consequently, is not likely to support good fish growth.

[35] Earlier stated that both highly acidic and alkaline medium (pH 5.50 to 6.50 and $\mathrm{pH} 9.0$ and above) is un- suitable for good fish production. [36-38] indicated that alkaline medium is a usual attribute of a productive water bodies. [36] and [39] reported the normal range for inland waters as 6.0 to 9.0 . The $\mathrm{pH}$ of Ohana Lake is within this range and hence not likely to constitute stressor for fish.

Generally, the minimum level of total alkalinity required for water to be most productive is $>50 \mathrm{ppm}$ [40]. The minimum level of total alkalinity preferred for fish culture is $100 \mathrm{mg} / \mathrm{L}$ [41]. The very low level of total alkalinity ( 20 to $25 \mathrm{mg} / \mathrm{L}$ ) in the lake is unlikely to support aquaculture growth.

Total hardness fell within WHO/EEC standard. [42] stated that a lake is adjudged to be distinctly eutrophic when the calcium exceeds $25 \mathrm{mg} / \mathrm{L}$. The only station (stn 5) with $25.9+0.001$ beyond this range was a small channel outside of the main lake but has some connection with the lake such that during high water level there is a mixing of the two water bodies.

[32] had mean conductivity in Lake Chad as $380.63 \pm$ 51.76. The very low electrolyte conductivity of the Lake which depicts low concentration of ions (i.e. charged solutes) compared to the range of 21.5 to $1523 \mu \mathrm{s} / \mathrm{cm}$ with a mean of $493.6 \pm 432.5 \mu \mathrm{s} / \mathrm{cm}$ in outdoor condition by [43] infers that Ohana Lake is unlikely to promote good aquaculture activity.

Turbidity is caused by wide variety of suspended solids, organic colloidal compounds and cause dispersion of sewage $[44,45]$. Turbidity reduces the light penetration and high rate of phytoplankton production is restricted to the upper waters. With water transparency using a secchi disc ranging from $0.8 \mathrm{~m}$ to $1.4 \mathrm{~m}$ with a mean of $1.0 \pm$ $0.28 \mathrm{~m}$, the secchi depth was seen to be inversely related to turbidity and determined the conditions of availability of light in the water column to support photosynthesis by phytoplankton, and hence primary production. The approximate vertical extent of the euphotic zone in Ohana Lake is $3.0 \mathrm{~m}$ following $[43,46]$ had a mean of $90.47 \pm$ 65.2 FTU, which promoted good fish culture.

The higher the silicate levels the more the diatoms. The low values are reflected in the low diatom group in the sample area.

The value for total dissolved solids in Ohana Lake is far below the upper limit set by [23] for polluted environment. Suspended solids or particulate matter in fresh or marine waters are of importance to aquaculture because they may damage fish gills and interfere with respiration. Secondly, they may cause siltation and smothering of benthos and interference with feeding of bivalve filter feeders. High turbidity due to suspended solids also reduce photosynthesis and hence production of phytoplankton and submerged periphyton. A high content of suspended organic solids will exert a biological oxygen demand and lead to oxygen depletion. Based on [23] 
guidelines the total dissolved solids in Ohana Lake are much less than the upper limit set that could cause pollution.

$\mathrm{BOD}_{5}$ levels usually depict the organic enrichment and show the decay of plants and animal matter in the Lake [47]. Water bodies with $\mathrm{BOD}_{5}$ higher than 35 - $45 \mathrm{mg} / \mathrm{L}$ are not to be considered as good quality for fish culture [48]. The $\mathrm{BOD}_{5}$ of Ohana Lake is comparatively quite low, and consequently, is not likely to contribute adversely to fish growth and culture. The minimum level of nitrate required for a lake to be productive is $0.1 \mathrm{mg} / \mathrm{L}$ [7]. The mean value of $0.645 \pm 0.54 \mathrm{mg} / \mathrm{L}$ in Ohana Lake is much higher than the minimum requirement and is likely to have contributed to the algal blooms observed in the lake.

The phosphate levels in the lake were zero in all the sampling stations. The apparent absence of phosphate may be due to the immediate utilization of nutrients by the phytoplankton. The absence of $\mathrm{PO}_{4}^{-} \mathrm{P}$ may also be due to the interaction between $\mathrm{PO}_{4}^{-} \mathrm{P}$ and calcium [49]. [50] indicated that the optimum level of phosphate needed for the growth of plankton is 0.1 to $0.2 \mathrm{mg} / \mathrm{L}$. When higher than this, may lead to eutrophication and algal blooms [51] and could be detrimental to fish. The present study has zero level phosphate concentration, which may be due to its utilization by plants to generate intense algal bloom. However these zero values are far below the optimum required for plankton growth required to form enough food for fish, and consequently, may not be able to boost the fish community of the Lake.

Highly coloured water is not desirable on aesthetic ground and may not be suitable for some industrial uses [52]. Apparent colour in Ohana ranged from $16 \mathrm{pt} / \mathrm{Co}$ to $45 \mathrm{pt} / \mathrm{Co}$ with a mean of $35.3 \pm 9.1 \mathrm{pt} / \mathrm{Co}$. At all sampling station the apparent colour in Ohana Lake is beyond the [23] standard of 7. These high colour values put Ohana Lake as a polluted aquatic medium.

[53] observed a total of 13 species of green algae, 14 species of blue-green algae and 24 species of diatoms in three water supply reservoirs in Tamilnadu.

The mean diversity index for the water flora of Ohana Lake was $0.969 \pm 2.55$ and $0.778 \pm 1.23$ for zooplankton. The equitability or evenness index $(\mathrm{J})$ yielded 1.61 and -3.05 , respectively, for phytoplankton and zooplankton. These low diversity index values indicate generally low species diversities as well as predominantly unstable ecosystem.

Fish yields from lakes and reservoirs are known to have strong correlation with primary production $[9,10]$. In aquaculture systems algal production, as measured by primary production, is also a good indicator of fish yields, although the relationship to fish yield is complicated by input of feed and other organic matter, such as manure. These may be ingested directly by the fish or channeled along the detritus pathway, thus boosting benthic production and indirectly the production of benthos feeding fishes. These values are far below the optimal levels 0.742 to $1.645 \mathrm{mgO}_{2} / \mathrm{L}$ (mean $0.771 \pm 0.509 \mathrm{mgO}_{2} / \mathrm{L}$ ) with average energy in joules of $28.108 \mathrm{j}$ to $60.788 \mathrm{j}$ (mean $30.886 \pm 20.05 \mathrm{j}$ ) as determined in fishponds by [43] suggesting that Ohana Lake is indeed oligotrophic.

When the light and dark bottles were kept beyond the photosynthetically active radiation, the initial DO became higher than the final DO and resulted into negative net primary production. Net photosynthesis decreased with depth.

A dimension to the problem of europhication is excessive growth of planktonic and attached algae and other aquatic macrophytes (water weeds), which exerts significant deleterious effects on the beneficial use of lakes [53]. [53] observed the presence of Oscillatoria sp., Anabaena sp. and Gomphonema sphaerophorum as the dominant form and concluded that the system was eutrophic. Similarly, the presence of these species including Microcystis sp and Aphonothece sp. in all the sampling stations in large amounts, in addition to three species of Pyrrophyceae, Glenodinium cinctum, Peridinium cinctum (O.F.M) and Gymnodinium excavatum often indicators of polluted environment must have contributed to the eutrophication of Ohana Lake. Potential and realized water quality deterioration is associated with excessive growth of algae and other aquatic plants, and alters the physicochemical factors of water [53]. Excessive growth due to eutrophication is known to release extra cellular products capable of acting as trihalomethanes (THM) precursors [54]. Also, Daum (USEPA, 1979-unpublished report, as cited by [54] reported that Anabaena and Pandorina morum serve as THM precursors when exposed to chlorination. [53] found Anabaena sp. as the chief component of algal community and frequently recorded algal blooms. One other factor that must have contributed to the pollution of the lake must have been the presence of aquatic weed Lemna minor in large amount around the Lake's small outlet. Finally, a look at the benthic community shows the dominance by the class Nemata. These nematode worms are often found in eutrophic bottoms.

The presence of diatoms Melosira sp. and Coscinodiscus excentricus are indications of polluted water bodies. Likewise Philodina sp (a Rotifera) is often associated with polluted waters.

The diversity index for the benthic flora was $0.371 \pm$ 0.57 while that of the benthic fauna was $2.23 \pm 2.80$. The equitability or evenness index ( $\mathrm{J}$ ) resulted in -15.50 and 4.18 , respectively, for benthic flora and fauna. The very low floral diversity index value indicates very low species diversities as well as predominantly unstable ecosystem while the faunal diversity index indicates slightly higher species diversity and a mild stable ecosystem. 
The high load of heavy metals in Ohana Lake must have been due mainly to anthropogenic activities emanating from accumulation of heavy metals from the explosives used during quarrying activities, sedimentation of rocks and car wash wherein car paints, oil and grease and metal were introduced into the system.

\section{Conclusions}

Residential development of lakeshores is expected to change a variety of key lake features that included: increased nutrient loading, increased invasion rate of nonnative species, increased exploitation rates of fishes by anglers, and alteration of littoral habitats. All of these factors may alter the capacity of lakes to support productive native fish populations [1]. Ohana Lake being quite close to residential area witnesses a lot of anthropogenic activities such as bathing; canoeing and car/motorcycle wash and consequently are prone to suffer this fate.

Both trace metals from the sediment and fish gills proved Ohana Lake to be a highly polluted aquatic environment. Furthermore, Ohana Lake is fast turning to a eutrophic ecosystem with accompanied algal bloom due to very high nutrient contents $\left(\mathrm{NO}_{3}\right.$ etc.). The planktonic community was dominated by algae, in particular, $\mathrm{Mi}$ crocystis spp, Oscillatoria sp., Aphanothece spp., Anabaena sp., Gomphonema sphaerophorum, Glenodinium cinctum, Peridinium cinctum (O.F.M) and Gymnodinium excavatum often known as indicators of aquatic pollution. The fish species observed during the sampling period were basically Tilapia galilea and Oreochromis niloticus and were of small sizes probably due to stress from pollution.

\section{Acknowledgements}

The author wishes to thank the Food and Agriculture Organisation (FAO) for providing the fund for carrying out this research.

\section{REFERENCES}

[1] D. E. Schindler, S. I. Geib and M. R. Williams. "Patterns of Fish Growth along a Residential Development Gradient in North Temperate Lake," Ecosystems, Vol. 3, No. 3, 2000, pp. 229-237. doi:10.1007/s100210000022

[2] S. Punita and S. C. Gajaria. "Feasibility and Utility of Green Algae for Metal Remedy," Indian Hydrobiology, Vol. 7, No. 1-2, 2004, pp. 85-88.

[3] A. A. Adebisi, "The Physicochemical Hydrology of a Tropical Seasonal River, Upper-Ogun River," Hydrobiologia, Vol. 79, No. 2, 1981, pp. 157-165. doi:10.1007/BF00006123

[4] A. M. A. Imevbore, "The Chemistry of the River Niger in the Kainji Reservoir Area," Arch. Hydrobiologia, Vol. 30, 1970, pp. 154-176.

[5] I. F. Adeniyi, "Studies of the Physicochemical Factors and the Planktonic Algae of Lake Kainji, Nigeria," Ph.D. Thesis, University of Ife, Ile-Ife, 1978.

[6] G. K. Iwama, M. M. Vijayan and J. D. Morgan, "The Stress Response in Fish," Ichthyology, Recent Research Advances, Oxford \& IBH Publishing Co., Pvt. Ltd., New Delhi, 2000.

[7] K. L. Sachidanandamurthy and H. N. Yajurvedi, "Monthly Variations of Water Quality Parameters (Physico-Chemical) of a Perennial Lake in Mysore City," Indian Hydrobiology, Vol. 7, No. 1-2, 2004, pp. 217-228.

[8] D. J. Forsyth and R. H. S. McColl, "Limnology of Lake Ngahewa, North Island N.Z," Journal of Marine and Freshwater Research, Vol. 9, No. 1, 1975, pp. 311-332.

[9] J. M. Melack, "Primary Productivity and Fish Yields in Tropical Lakes," Transactions of the American Fisheries Society, Vol. 105, No. 5, 1976, pp. 575-580. doi:10.1577/1548-8659(1976)105<575:PPAFYI $>2.0$.CO; $\underline{2}$

[10] T. R. Oglesby, "Relationship of Fish Yield to Lake Phytoplankton Standing Crop, Production and Morphoedaphic Index," Journal of the Fisheries Research Board of Canada, Vol. 34, No. 12, 1977, pp. 2271-2279. doi:10.1139/f77-305

[11] G. Idodo-Umeh, "Contaminations of Heavy Metals in Water and Hemichromis fasciatus of a Waste Pit Influenced by Petroleum Activities," Tropical Freshwater Biology, Vol. 16, No. 2, 2007, pp. 45-56.

[12] H. P. Stirling, "Chemical and Biological Methods of Water Analysis for Aquaculture," University of Stirling, Stirling, 1985.

[13] H. I. Henderson, "The Significance of Limnology in the Development of Fisheries in Man-Made Lake and RiverBasins," The Proceedings of the International Conference of Kainji Lake and River Basins Development in Africa, Ibadan, 11-17 December 1977.

[14] M. Holden and W. Reed, "West African Freshwater Fish,” Longman Group, London, 1978.

[15] G. E. Beedham, "Identification of the British Mollusca," Pitman Press, Bath, 1972.

[16] H. Maochlan, "Illustration of Freshwater Plankton," China Press, Kuala Lumpur, 1983.

[17] J. G. Needham and P. R. Needham, "A Guide to the Study of Freshwater Biology," 5th Edition, Holden-Day, Inc., San Francisco, 1984.

[18] C. Y. Jeje and C. F. Fernando, "A Practical Guide to the Identification of Nigerian Zooplankton (Cladocera, Copepoda and Rotifera)," KLRI, New Bussa, 1986.

[19] R. A. Vollenweider, "A Manual on Methods for Measuring Primary Production in Aquatic Environments," IPB Handbook No. 12, 3rd Edition, Oxford Blackwell, Oxford, 1985.

[20] R. Rump and R. Krist, "Manual for the Laboratory Examinations of Water, Wastewater and Soil," 1987.

[21] T. R. Parsons, Y. Maita and C. M. Lalli, "Manual of Chemical and Biological Methods for Seawater Analysis," Pergamon Press, Oxford, 1984.

[22] APHA, "Standard Methods for the Examinations of Wa- 
ter and Wastewater," 19th Edition, America Public Health Association, Washington DC, 1995.

[23] FEPA, "Federal Environmental Protection Agency. S.1.9 National Interim Guidelines and Standards for Industrial Effluents, Gaseous Emissions and Hazardous Waste Management in Nigeria," 1991.

[24] World Health Organisation, "Guidelines for Drinking Water Quality,” WHO, Geneva, 1984.

[25] World Health Organisation, "Environmental Health Criteria, No. 10: Principles for Safety Assessment of Food Additives and Contaminants in Food," WHO, Geneva, 1987.

[26] W. Chen, S. K. Tan and J. H. Tay, "Distribution, Fractional Composition and Release of Sediment-Bound Heavy Metals in Tropical Reservoirs," Water, Air \& Soil Pollution (Historical Archive), Vol. 92, No. 3-4, 1996, pp. 273287.

[27] E. K. Ajani and S. O. Ayoola, "The Heavy Metals in the Muscle of Some Imported Frozen Fish in Ibadan, Nigeria," Journal of Environmental Extension, Vol. 6, 2007, pp. 1-4. doi:10.4314/jext.v6i1.2753

[28] S. E. Kakulu and O. Osibanjo, "A Baseline Study of Mercury in Niger Delta Area of Nigeria," Environmental Pollution, Vol. 11, No. 4, 1986, pp. 315-322. doi:10.1016/0143-148X(86)90048-0

[29] H. A. Hervey and C. Lee, "Hydrology and Plankton of Eleiyele Reservoir," Hydrobiologia, Vol. 30, 1982, pp. 154-176.

[30] O. G. Adeyemi, G. O. Adediran and T. Oyeniyi, "Some Trace Elements Concentration in Variety of Fishes from Asa River, Ilorin, Nigeria," Bioscience Research Communications, Vol. 8, No. 2, 1996, pp. 99-102.

[31] I. A. Amoo, O. T. Adebayo and A. J. Lateef, "Evaluation of Metals in Fishes, Water and Sediments of Lake Kainji," Nigerian Journal of Food, Agriculture and Environment, Vol. 3, No. 1, 2005, pp. 209-212.

[32] S. N. Umeham, "Some Aspects of the Physicochemical limnology of Lake Chad (Southern Sector)," Journal of Aquatic Sciences, Vol. 4, 1989, pp. 21-26.

[33] A. K. Gupta and R. S. Mehrotra, "Studies on Seasonal Variation in $\mathrm{pH}$ and DO Content in Senthil Sarovar, Kurukshetra," Geobios, Vol. 13, No. 6, 1986, pp. 276-278.

[34] L. R. Bhatt, P. Lacoul, H. D. Lekhak and P. K. Jha, "Physico-Chemical Characteristics and Phytoplankton of Taudaha Lake, Kathmandu," Pollution Research, Vol. 18, No. 4, 1999, pp. 353-358.

[35] S. M. Banerjea, "Water Quality and Soil Condition of Fishponds in Some States of India in Relation to Fish Production," India Journal of Fisheries, Vol. 14, No. 1-2, 1967, pp. 115-144.

[36] G. E. Hutchinson, "A Treatise on Limnology. Geography, Physics and Chemistry," John Wiley \& Sons Inc., New York, 1957.

[37] S. B. Saxena and A. D. Adoni, "Diurnal Variation in Sagar Lake, Sagar (India). 1. Studies in Deep Water Areas," Hydrobiologia, Vol. 37, No. 1, 1973, pp. 7-31.

[38] S. Ayyappan and T. R. Gupta, "Limnology of Ramsamdra Tank-Hydrograph, Mysore," Journal of Agricultural
Science, Vol. 15, 1981, pp. 305-312.

[39] P. S. Welch, "Limnology," McGraw-Hill Book Co. Inc., New York, 1952.

[40] W. Ohle, "Chemisch-Stratigraphische Untersuchungen der Sediment-Metamosphozeeines eines Waldsees," Biochemische Zeitschrift, Vol. 258, 1993, pp. 420-428.

[41] G. L. Shroeder, "Fish Farming in Manure Loaded Ponds," Proceedings ICLARMSEARCA Conference in Integrated Agriculture and Aquaculture Farming Systems, ICLARM Proceedings, Vol. 4, 1980, pp. 73-86.

[42] O. M. Lind, "Handbook of Common Methods in Limnology," The C.V. Mosby Company, London, 1979.

[43] P. O. Ajah, "Identification and Mass Production of Zooplankton Species Suitable for Early Feeding of African Clariid Catfish," Ph.D. Thesis, University of Calabar, Calabar, 1995.

[44] A. A. Agrawal, "Induced Responses to Herbivory in Wild Radish: Effects on Several Herbivores and Plant Fitness," Ecology, Vol. 80, No. 5, 1999, pp. 1713-1723. doi:10.1890/0012-9658(1999)080[1713:IRTHIW]2.0.CO; $\underline{2}$

[45] H. C. Kataria, "Preliminary Study of the Drinking Water of Piparian Township," Pollution Research, Vol. 19, No. 4, 2000, pp. 645-649.

[46] R. C. Hart and C. Boane, "Limnology of Southern African Coastal Lakes-New Vistas from Mozambique," African Journal of Aquatic Science, Vol. 29, No. 2, 2004, pp. 145-159. doi:10.2989/16085910409503806

[47] K. F. Anil and M. Musaddiq, "A Study on PhysicoChemical Characteristic of Kapshi Lake and Purna River Waters in Akola District of Maharastra (India)," Nature Environment and Pollution Technology, Vol. 1, No. 3, pp. 261-263.

[48] K. S. Pande and S. D. Sharma, "Studies on Water Quality Index for Ramaganga River at Moradabad, UP," Pollution Research, Vol. 18, No. 3, 1999, pp. 327-333.

[49] S. Rajan, "Biology of Madras Drinking Water Supply System and Health," Ph.D. Thesis, University of Madras, Madras, 1986.

[50] A. Screenivasan, "Limnology and Productivity of Tropical Upland Impoundments in Nilgiris, Madras States India," Phytos, Vol. 7, No. 1, 1965, pp. 146-160.

[51] C. N. Sawyer, "Fertilization of Lake by Agriculture and Urban Drainage," Journal of New Water Lakes Association, Vol. 51, 1947, pp. 109-127.

[52] I. U. Ubong and A. E. Gobo, "Fundamentals of Environmental Chemistry and Meteorology," Tom \& Harry Publications Ltd., 2004.

[53] S. Rajan and J. Azariah, "Seasonal Changes of Planktons in the Three Water Supply Reservoirs of Madras Drinking Water Supply, Tamilnadu-A Public Health Approach," Indian Hydrobiology, Vol. 7, No. 1-2, 2004, pp. 205-215.

[54] J. M. Symons, A. A. Stevens, R. M. Clark, E. E. Geldreich, D. T. Love and J. Demarco, "Treatment Techniques for Controlling Trihalomethanes in Drinking Water," US Environmental Protection Agency, Cineinnati, 1981. 